\title{
Investment horizons : A time-dependent measure of asset performance
}

\author{
Ingve Simonsen ${ }^{1}$, Anders Johansen ${ }^{2}$, and Mogens H. Jensen ${ }^{3}$ \\ 1 Department of Physics, NTNU, NO-7491 Trondheim, Norway \\ 2 Teglgårdsvej 119, DK-3050 Humlebæk, Denmark \\ 3 Niels Bohr Institute, Blegdamsvej 17, DK-2100 Copenhagen Ø, Denmark
}

Summary. We review a resent time-dependent performance measure for economical time series - the (optimal) investment horizon approach. For stock indices, the approach shows a pronounced gain-loss asymmetry that is not observed for the individual stocks that comprise the index. This difference may hint towards an synchronize of the draw downs of the stocks.

As an investor or practitioner working in the financial industry, you are continuously faced with the challenge of how to chose and manage a portefolio under varying market conditions; as the market change, you have to decide whether to adjust your positions in order to make the portfolio, as you see it, more optimal. The way such important decisions are made, with dramatic economic consequences if done badly, is rather complex; most market players have their very own methods for this purpose, and they are only rarely disclosed to the public. The clients risk aversion, which is based on individual psychology, plays a major role in the task of choosing a portfolio and hence quantifiable and rational measure must be used in for example stress testing of the portfolio.

As the financial industry became fully computerized, the distribution of returns approach became popular for measuring asset performance from historic data records. Today, this method is considered one of the classic approaches for gauging the performance of an asset $[1,2]$. The method relies on the distribution of returns (to be defined below) corresponding to a fixed time window (or horizon as we will refer to it below). In order to look into the performance over a different time horizon, the return distribution has to be regenerated for the new window size. Actually, one of the most successful strategies for actively investing when the risk aversion is not low, is to, a priori, decide for a return level and then liquidate the position when this level has been reached.

It is not at all clear that the natural scenario for an investor is to consider fixed time windows. There has therefore lately been a lot of interest in time dependent measures, i.e. measures where the time period over which the asset is hold, is non-constant, and allowed to depend on the specific market condi- 
tions which in general is not known in detail. A change in time horizon used by an investor may be due to for instance a changes in the market itself, or new investment strategies being implemented by the investor.

In this work, we will review a recent development in such time-dependent measures - the investment horizon approach. This approach is motivated by progress in turbulence [3], and it represents an adaption of a more general concept, known as inverse statistics, to economics. The investment horizon approach was first introduced into economics by the present authors [4], and later considered in a series of publications $[5,6,7,8]$. The method has recently been applied to different types of financial data with success; stock index data $[4,5,6]$, like the Dow Jones Industrial Average (DJIA), NASDAQ, Standard and Poor 500 (SP500), individual stocks [8], and high frequency foreign exchange (FX) data [6]. A similar approach, however without a fixed return level, has been studied in Refs. $[9,10]$ with the prime focus on losses.

Let $S(t)$ denote the asset price, and $s(t)=\ln S(t)$ the corresponding logarithmic price. Here time $(t)$ can be measured in different ways [2], but the various choices may result in different properties for the inverse statistics [6]. The logarithmic return at time $t$, calculated over a time interval $\Delta t$, is defined as $[1,2] r_{\Delta t}(t)=s(t+\Delta t)-s(t)$.

We consider a situation where an investor is aiming for a given return level denoted by $\rho$. This level may be both positive (gains) or negative (losses). If the investment is made at time $t$, then the investment horizon is defined as the time $\tau_{\rho}(t)=\Delta t$ so that the inequality $r_{\Delta t}(t) \geq \rho\left(r_{\Delta t}(t) \leq \rho\right)$ for $\rho \geq 0$ $(\rho<0)$ is satisfied for the first time. In mathematical terms, this can be expressed as

$$
\tau_{\rho}(t)= \begin{cases}\inf \left\{\Delta t \mid r_{\Delta t}(t) \geq \rho\right\}, & \rho \geq 0, \\ \inf \left\{\Delta t \mid r_{\Delta t}(t) \leq \rho\right\}, & \rho<0 .\end{cases}
$$

The investment horizon distribution, $p\left(\tau_{\rho}\right)$, is then the distribution of investment horizons $\tau_{\rho}$ estimated from the data (cf. Fig. 1a). This distribution will go through a maximum, as should be apparent from the discussion to follow. This maximum - the optimal investment horizon - will be denoted $\tau_{\rho}^{*}$. It quantifies the most likely time period (obtained from historic data) needed to reach the investment outcome characterized by $\rho$.

For later use, we stress that if the price process $S(t)$ is a geometrical Brownian motion - the classic assumption made in theoretical finance - then the solution to the investment horizon (first passage time) problem is known analytically [11]. It can be shown that the investment horizon distribution is given by the Gamma-distribution: $p(t)=|a| \exp \left(-a^{2} / 2 t\right) /\left(\sqrt{2 \pi} t^{3 / 2}\right)$, where $a \propto \rho$. Hence, in the limit of large (waiting) times, one recovers the well-known first return probability $p(t) \sim t^{-3 / 2}$.

Figures 1 show empirical investment horizon distributions, $p\left(\tau_{\rho}\right)$ with $\rho=$ \pm 0.05 . for an index (Fig. 1a) and an individual stock (Fig. 1b). Drift-terms that were "smooth" up till a time scale of roughly 4 years, were removed from the logarithmic prices prior to the analysis (consult Ref. [4] for details). 

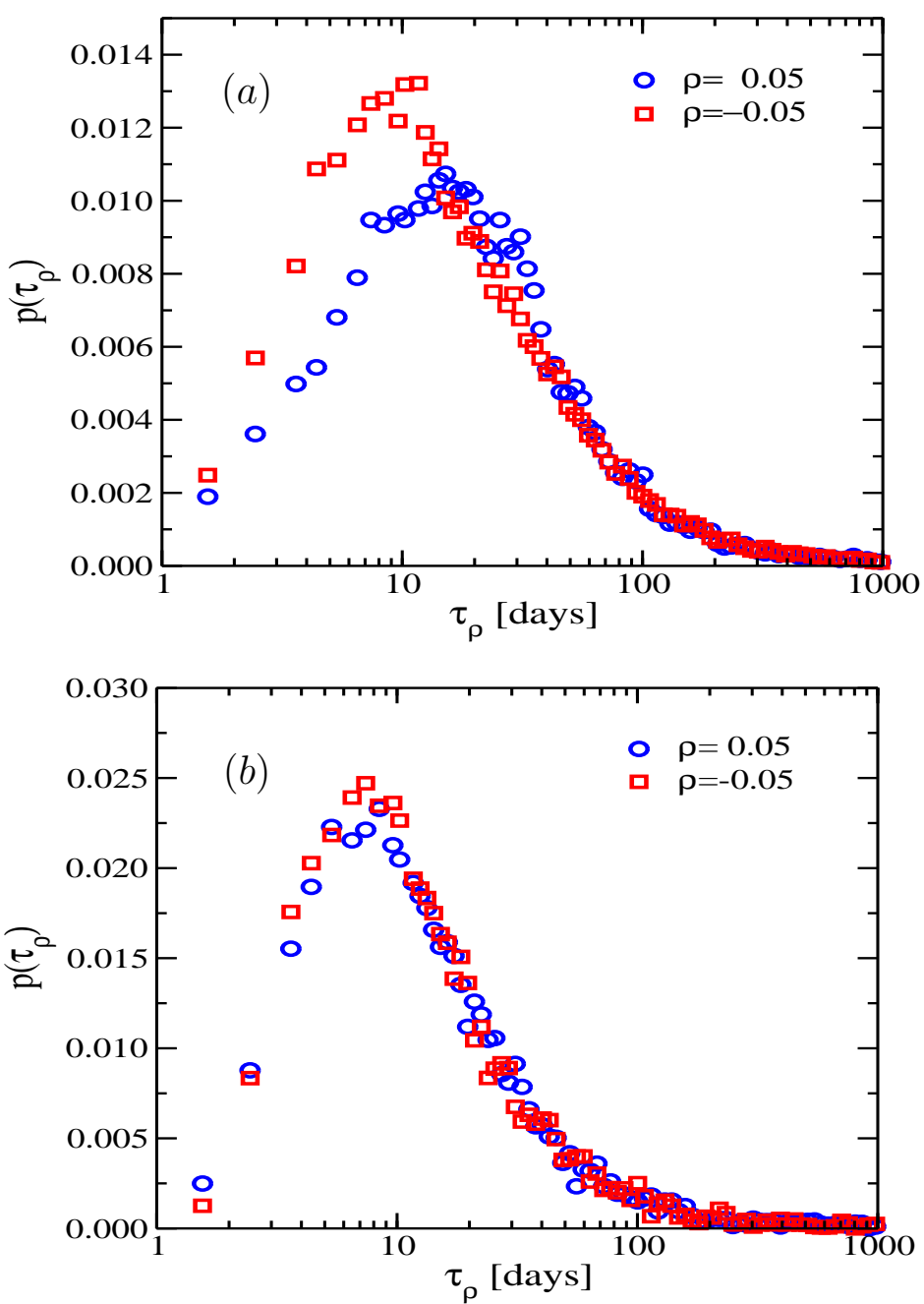

Fig. 1. (a) The investment horizon distributions of the DJIA closing prices from 1896 till present, at a return levels $|\rho|=0.05$. (b) The same as Fig.1(a), but now for the single stock of IBM for the period from the beginning of 1962 till June 2000. IBM has been part of DJIA since June 29, 1979.

This pre-processing of the data was done in order to enable a more consistent comparison of the results corresponding to positive and negative levels of returns due to differences in economic fundamentals such as inflation, interest rates, etc. The data set used to produce the results of Fig. 1a was the daily close of the Dow Jones Industrial Average taken over its whole history up till present. From this same figure, two well-pronounced, but not coinciding, optimal investment horizons can be observed from the empirical distributions 


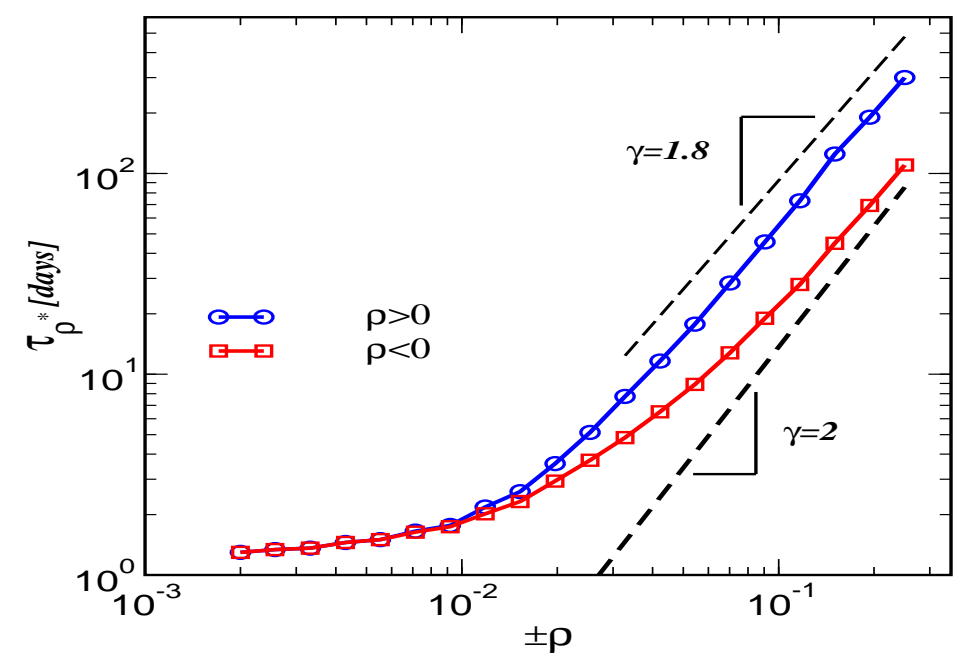

Fig. 2. The optimal investment horizon $\tau_{\rho}^{*}$ for positive (open circles) and negative (open squares) levels of return $\pm \rho$. In the case $\rho<0$ one has used $-\rho$ on the abscissa for reasons of comparison.

$p\left(\tau_{\rho}\right)$. With $|\rho|=0.05$ they are both of the order of $\tau_{\rho}^{*} \sim 10$ days. In general, the values of $\tau_{\rho}^{*}$ will depend on the return level $\rho$, and we presents results for the DJIA in Fig. 2 for positive and negative return levels. Recall that if the price process is consistent with a geometrical Brownian motion, one has $\tau_{\rho}^{*} \sim \rho^{\gamma}$ with $\gamma=2$ for all values of $\rho$ (lower dashed line in Fig. 2). The empirical results are observed not to be consistent with such a behavior. For rather small levels of returns - a fraction of a percent - the dependence on return level is quite weak. When $|\rho|$ is increased, however, the dependence becomes more pronounced and it gradually becomes more and more like, but still different from, the geometrical Brownian result. As a whole, the dependence of $\tau_{\rho}^{*}$ (on $\rho$ ) over the range of return levels considered in Fig. 2, resembles more a double logarithmic behavior than a power law. However, for the range of $\rho$-values considered and the fact that the statistics become poorer for increasing levels of return, we are unable from the empirical data alone to uncover the actual functional dependence of $\tau_{\rho}^{*}$ on the return level.

One of the most striking features of Fig. 2 is the apparent fact that the optimal investment horizon for positive and negative return levels are not the same. This asymmetry starts to develop when the return level $|\rho|$ is not too small (cf. Fig. 2). Such a gain-loss asymmetry is actually a rather general feature of the investment horizon of stock indices [6]. On the other hand, for individual stocks that together comprise the index, this phenomenon is less pronounced [8] and an asymmetry can often hardly be seen at all. In Fig. 1b this is exemplified by the investment horizons of IBM for $\rho= \pm 0.05$, a company that is part of the DJIA index. Similar results hold for most other stocks 
being part of the DJIA [8]. The attentive reader could ask: How is it possible that an asymmetry is present in the index, but not in the individual stocks that together make out the index? At the time of writing, there is no consensus on what is causing this behavior. It has been speculated that it might be caused by cooperative effects taking place among the stocks and causing them to partly synchronize their draw-downs (conditional serial correlation). If that was to be the case, the index - that is some average of the individual stocks will experience an increased probability of shorter investments horizons $\tau_{-|\rho|}$ compared to the similar results found for the same positive level of return. Other speculations go in the direction of this phenomenon being related to the so-called leverage effect [12]. These questions are being addressed by ongoing research efforts, and it is hoped that they will be satisfactory answered in the immediate future.

Before ending this contribution, we would like to add a few comments regarding possible practical implications (as we see it) of the investment horizon approach [13]. Two applications will be mentioned here, both taken from portfolio management. The first application is related to the problem of consistent allocation of VAR-like (quantile) and stop-loss limits. For such problems, the correlation structure over different time horizons is important. Our approach naturally use non-fixed time windows, and it is therefore hoped that it might contribute some new insight onto these issues. The second application is concerned with the calculation of risk measures for portfolios. When the market is moving against you, you are forced to liquidate. In this process, "liquidation horizons" that are used across assets of a portfolio, are normally not the same. By taking advantage of the negative return levels, investment horizon distributions $p\left(\tau_{-|\rho|}\right)$ for the different assets of the portfolio, may be used to design an optimal liquidation procedure depending on the nature of the position, e.g., long or short. The exploration of possible applications of the concept of inverse statistics in economics is at its infancy. We hope that the future will demonstrate this approach to be fruitful also from a practical standpoint.

A new measure of asset performance that represents an alternative to the classic distribution of returns approach has been described. Unlike the classic

method, the new technique is time-dependent. This opens the possibility of studying and measure asset performance over a non-constant time scale, an idea that lately has attracted a great deal of attention.

\section{Acknowledgements}

The first author wishes to thank Drs. Ted Theodosopoulos and Marc Potters for fruitful discussions and valuable comments and suggestions. IS also acknowledges the financial support kindly provided by Nihon Keizai Shimbun Inc. 


\section{References}

1. J.-P. Bouchaud and M. Potters. Theory of financial risks : from statistical physics to risk management. Cambridge University Press Cambridge 2000.

2. R. N. Mantegna and H. E. Stanley. An Introduction to Econophysics: Correlations and Complexity in Finance. Cambridge University Press Cambridge 2000.

3. M.H. Jensen. Multiscaling and Structure Functions in Turbulence: An Alternative Approach. Phys. Rev. Lett. 83, 76 (1999).

4. Ingve Simonsen, Mogens H. Jensen and Anders Johansen. Optimal Investment Horizons. Eur. Phys. J. B 27, 583 (2002).

5. M.H. Jensen, A. Johansen and I. Simonsen. Inverse Fractal Statistics in Turbulence and Finance. Int. J. Mod. Phys. B 17, 4003 (2003).

6. M.H. Jensen, A. Johansen and I. Simonsen. Inverse Statistics in Economics : The gain-loss asymmetry. Physica A 324, 338 (2003).

7. M.H. Jensen, A. Johansen, F. Petroni and I. Simonsen. Inverse Statistics in the Foreign Exchange Market. Physica A 340, 678 (2004).

8. A. Johansen, I. Simonse and M.H. Jensen. Unpublished work. 2004.

9. A. Johansen and D. Sornette. Stock market crashes are outliers. Eur. Phys. J. B 1, 141 (1998).

10. A. Johansen and D. Sornette. Large stock market price drawdowns are outliers. J. of Risk 4 (2), 69 (2001/2002).

11. D. Kannan. Introduction to Stochastic Processes. North Holland New York 1979.

12. J.-P. Bouchaud, A. Matacz and M. Potters. Leverage Effect in Financial Markets: The Retarded Volatility Model. Phys. Rev. Lett. 87, 228701 (2001).

13. Ted Theodosopoulos. Private communication. 2004. 